\title{
LE DÉCRET ACCOMPAGNANT LE RÈGLEMENT DE TOMIS SUR LA VENTE DE LA PRÊTRISE DES DIEUX DE SAMOTHRACE $($ LGS II $84=$ I. TOMIS 1)
}

\author{
Alexandru Avram - Dragoş Hălmagi \\ Le Mans Université - Institut d'archéologie «Vasile Pârvan » (Bucarest) \\ alexandru.avram@univ-lemans.fr - $\underline{\text { dragoshhalmagi@gmail.com }}$
}

\section{RÉSUMÉ}

Les auteurs proposent une tentative de restauration de ce décret fragmentaire, en supposant que le Conseil et le peuple de Tomis décida d'honorer Timée, fils de Stratôn, l'homme qui également acquit le sacerdoce des Dieux de Samothrace. Ils proposent également que les deux textes furent inscrits en même temps, vers 100 a.C.

MOTS-CLÉ: Décret, vente du sacerdoce, restauration, Tomis, les Grands Dieux de Samothrace.

\section{THE DECREE ACCOMPANYING THE TOMITAN REGULATIONS \\ CONCERNING THE SALE OF THE PRIESTHOOD OF THE SAMOTHRACIAN GODS \\ $($ LGS II $84=$ I. TOMIS 1)}

\section{ABSTRACT}

The authors suggest a tentative restoration of this fragmentary decree, assuming that the Council and the People of Tomis decided to honour Timaios, son of Straton, the man who also purchased the priesthood of the Samothracian Gods. They also suggest that both texts were inscribed at the same time, around $100 \mathrm{BC}$.

KeYwords: Decree, priesthood sale, restoration, Tomis, Great Gods of Samothrace.

Le règlement concernant la vente de la prêtrise des Dieux de Samothrace est connu depuis longtemps. Publié concurremment par G. Tocilescu et D. Markopoulos $^{2}$, il fut réédité dans leurs recueils de lois sacrées par L. Ziehen ${ }^{3}$, ensuite par F. Sokolowski ${ }^{4}$, et finalement par I. Stoian dans son corpus des inscriptions de Tomis ${ }^{5}$ et commenté à plusieurs reprises - avec parfois de nouvelles suggestions de lecture et de restitution - depuis le lendemain de sa publication jusque dans les dernières années ${ }^{6}$.

Ce règlement, dont le texte est conservé de manière assez satisfaisante - seules quelques lacunes continuant à poser problème - est inscrit comme colonne II (à droite) d'une plaque de marbre blanc (Fig. 1) brisée en haut et en bas (dimensions en mètres : 
ht. 0,34 , lg. 0,44, ép. 0,10 ; lettres soigneusement gravées, ht. 0,007-0,01 à gauche et 0,008-0,013 à droite; interligne 0,004-0,006 à gauche et 0,005-0,007 à droite) déposée jadis au Musée national des antiquités (actuellement Institut d'archéologie "Vasile Pârvan » de Bucarest, inv. $n^{\circ}$ L 922) et transférée ensuite au Musée national d'histoire de Roumanie de la même ville (inv. $\mathrm{n}^{\circ}$ 18817) :

$$
\text { col. II }
$$

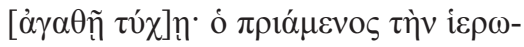

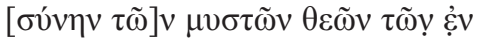

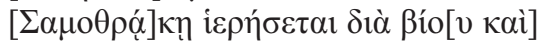

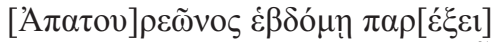

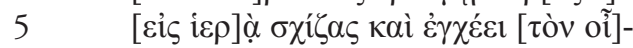

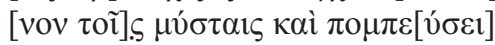

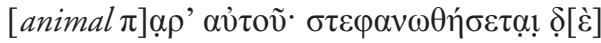

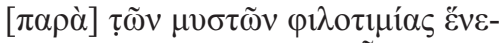

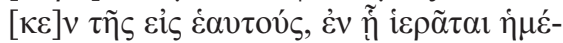

10

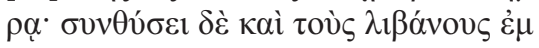

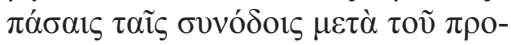

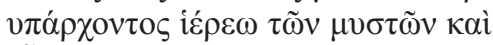

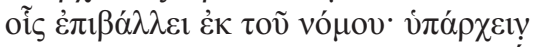

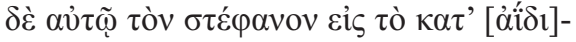

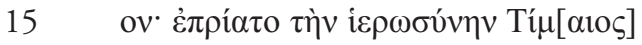

$\Sigma \tau \rho \alpha \dot{\tau} \tau \omega v o \varsigma$ $\chi \rho v \sigma \tilde{\omega} v \dot{\varepsilon} \pi \tau \dot{\alpha}, \chi \alpha \lambda \kappa[\tilde{\omega} v]$

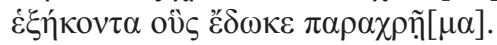

vacat

${ }^{1}$ Tocilescu, 1882 : 8-9, $\mathrm{n}^{\circ} 14$, transcription en majuscules, avec les restitutions de Th(eodor) G(omperz). Tocilescu attribuait faussement l'inscription à Callatis, ce qui est démenti non seulement par les traits de langue (pas de dorismes), mais surtout par la mention précise de l'autre éditeur (voir ci-dessous), qui inclut ce monument dans son recueil d'inscriptions de Tomis. Voir, à ce propos, Robert, 1935-1936.

${ }^{2}$ Markopoulos, 1884-1885: 47, n 419, et d'après cette édition, Michel, $1900: \mathrm{n}^{\circ} 704$, qui ne reprend que la colonne droite, celle contenant le règlement.

${ }^{3}$ LGS II 84. Voir aussi Danoff, $1932: 1-11, \mathrm{n}^{\circ} 1$.

${ }^{4}$ Sokolowski, 1969 : 173-175, nº 87 (SEG 25, 755), seule la colonne droite, et d'après cette édition, Cole, 1984 : 142-143, $\mathrm{n}^{\circ} 6$.

${ }^{5}$ I. Tomis 1 , avec photo. Comme travail préparatoire annonçant le futur corpus, voir aussi Stoian, $1962: 75-78, \mathrm{n}^{\circ}$ I.1, avec photo (SEG 24, 1047). Lédition de Stoian fut ensuite reprise sans changements dans Bâltâc - Ştirbulescu - Ştefan, $2015: 50, n^{\circ} 31$, avec photo. Voir aussi, avec quelques compléments de lemme, I. Tomis Suppl. 1, avec photo.

${ }^{6}$ Herbrecht, $1885: 8-9.32 .34 .45$; Ziebarth, $1896: 57, n^{\circ} 1.207$; Rubensohn, $1898: 292$; Poland, $1909: 557, \mathrm{n}^{\circ}$ B96 (voir aussi p. 37, avec n. **. 224. 263. 267. 341, n. *. 350. 418. 420. 426, n. ††. 433-434. 510. 528) ; Kern, 1919 : 1414 ; Pârvan, 1924 : 277 ; Roussel, 1926 : 313-317 ; Robert, 1935-1936 (cf. J. et L. Robert, Bull. ép. 1938, 237) ; Hemberg, 1950 : 222-223 ; Nilsson, 1955 : 48, n. 2. 58 ; Pippidi, $1962: 140$, n. 1 [= Pippidi, $1969: 56$, n. 76] ; Robert, $1966: 199$ [= Robert, 1990 : 623], n. 150 ; Pippidi, 1967 [= Pippidi, 1988 : 224-225] ; Parker, Obbink, 2000 : 421-422, n. 16, et 424-426; Chiekova, $2008: 204$. 


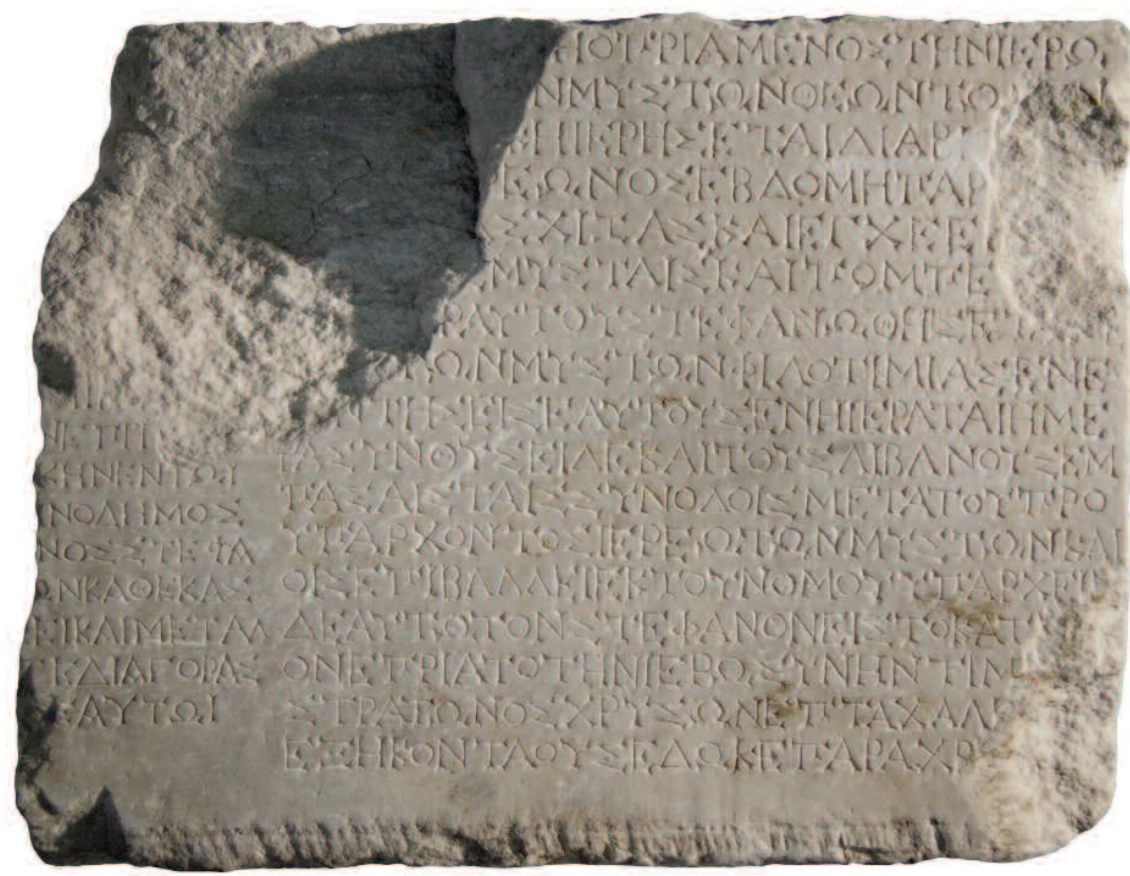

Fig. 1. Inscription I. Tomis 1. Photo George Nica, (C) Musée national d'histoire de Roumanie.

Restitutions dues pour la plupart à Gomperz apud Tocilescu, 1882 (Marko-

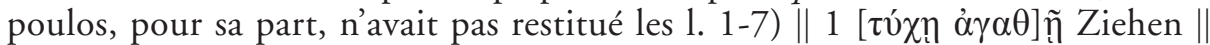

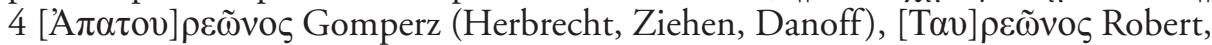

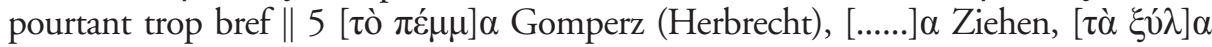

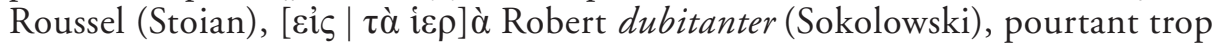

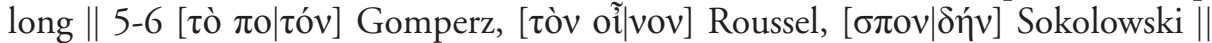

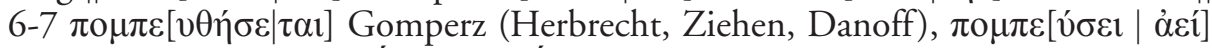

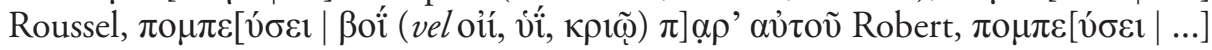

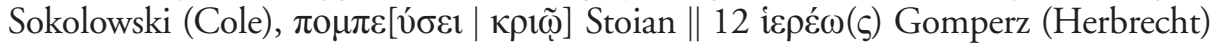

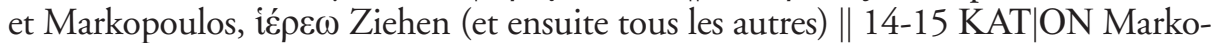

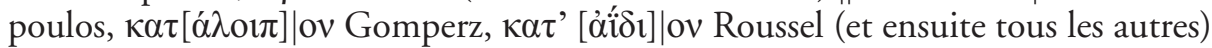
\|| $17 \pi \alpha \rho \alpha \chi[-$ Markopoulos, $\pi \alpha \rho \alpha \chi \rho \tilde{\eta}[\mu \alpha]$ Gomperz.

Nous ne saurions nous attarder à commenter ici ce règlement ${ }^{7}$. En revanche, la colonne I de la stèle (celle gravée à gauche et conservée d'une manière décourageant

${ }^{7}$ Nous nous contentons de renvoyer pour $\sigma \chi i \zeta \zeta \alpha$, « bûchettes » (1. 5), à Robert, $1966: 199$ [= Robert, $1990: 623$ ], n. 150, et plus récemment, à Lupu, 2009: 169. 224, avec de nombreux exemples

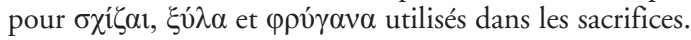


à la première vue tout essai de restitution) - ce qui explique peut-être pourquoi elle a moins excité la sagacité des savants - fera désormais l'objet de notre intervention. Nous commençons par reprendre l'édition figurant dans I. Tomis 1 :

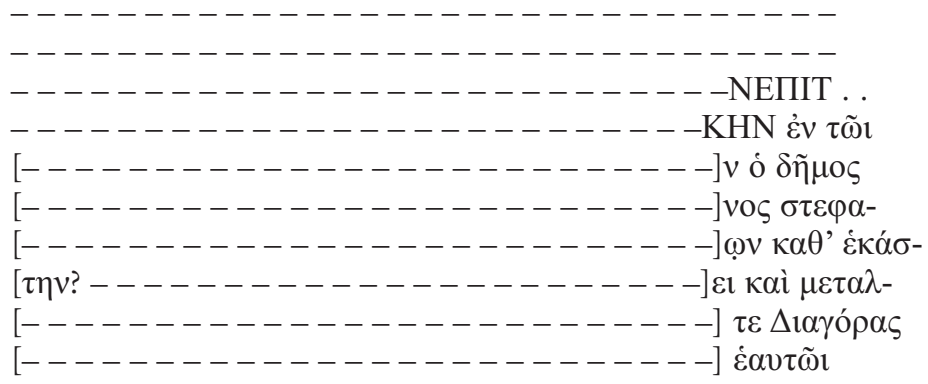

9 NB Gomperz (Ziehen, Danoff), lettres mentionnées dans l'apparat critique, pourtant non transcrites par Stoian $\| 10-] v \dot{\varepsilon} \pi \imath \pi[\ldots .$.$] Gomperz (Ziehen, Danoff),$

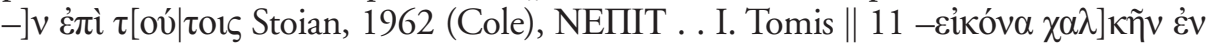

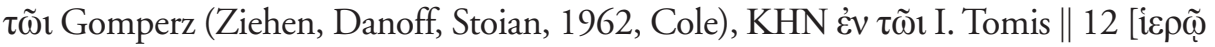

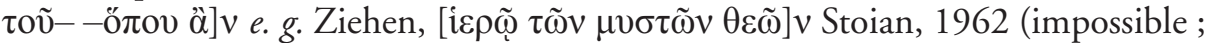

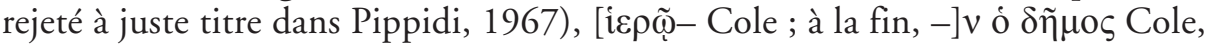

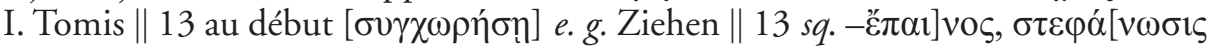

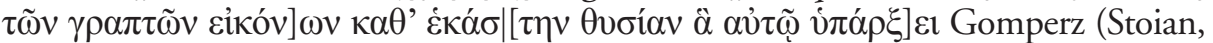

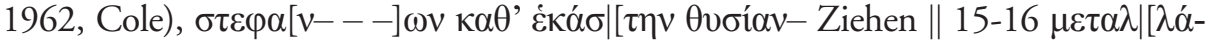

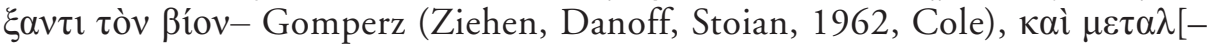

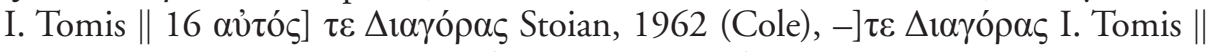
$17 \dot{\varepsilon} \alpha v \tau \tilde{\omega} \iota$ Gomperz (Stoian, Cole), $\dot{\varepsilon} \alpha v \tau \tilde{\omega}$ i- Ziehen.

Tout d'abord, quelques observations préliminaires.

Les deux colonnes sont écrites avec des lettres de tailles différentes : les lettres de la colonne I sont plus petites que les lettres de la colonne II. La ligne 9 de la colonne de gauche se situe entre les lignes 9 et 10 de la colonne de droite, il s'agit donc en fait de la ligne 10.

La forme des lettres est presque la même : seul l'alpha est différent, à barre brisée dans la colonne I, à barre droite dans la colonne II. De surcroît, le deuxième texte ne note plus l'iôta anekphônèton. La différence d'orthographe a été interprétée comme une preuve d'une longue période de temps écoulée entre les gravures des deux documents ${ }^{8}$, mais cette oscillation n'est pas rare dans les inscriptions hellénistiques et d'époque impériale dans le monde grec, parfois dans le même texte?

\footnotetext{
${ }^{8}$ Stoian, $1962: 78$, avec des arguments repris dans le commentaire à I. Tomis 1.

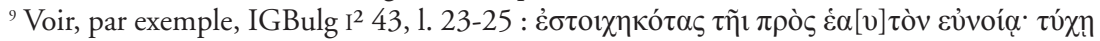
$\dot{\alpha} \gamma \alpha \theta \tilde{n} \delta \varepsilon \delta o ́ \chi \theta \alpha \imath \tau \tilde{\omega} 1 \delta \eta ́ \mu \omega t$.
} 
la disposition soignée des deux textes indique que le deuxième était connu au moment où le premier a été gravé.

L. 9. Nous distinguons vers la fin de la ligne les lettres Hị, ce qui encourage la restitution $\tau] \tilde{\eta} 1 \beta[$ ov $\lambda \tilde{\eta}$.

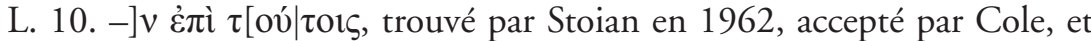
abandonné par le même Stoian dans son édition du corpus, nous semble s'imposer,

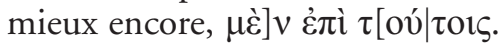

L. 11. Nous ne voyons aucun encombre majeur pour accepter la solution déjà trouvée jadis par Gomperz et adoptée par plusieurs éditeurs, [Eiંóv $\chi \alpha \lambda] \kappa \tilde{v} v$. Dans ce dessein, après $\dot{\varepsilon} v \tau \tilde{\omega}$, on attendrait à la ligne suivante la désignation de l'endroit, très probablement un sanctuaire, où devait être exposée cette statue en bronze.

L. 12. Après la lacune et avant la lettre $\mathrm{N}$ lue par nos prédécesseurs, on peut distinguer un trait vertical.

L. 15-16. Un simple regard dans les dictionnaires, afin de repérer les mots commençant par $\mu \varepsilon \tau \alpha \lambda$ - et qui conviendraient ici comme sens, suffit pour constater qu'il n'y a pas d'autre solution possible que celle proposée dès le début par Gomperz et acceptée par d'autres aussi (y compris par Stoian en 1962), donc $\mu \varepsilon \tau \alpha \lambda \mid\left[\lambda \alpha{ }^{\prime} \xi \alpha v \tau 1\right.$

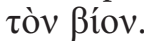

L. 16 : Contrairement à nos prédécesseurs, nous lisons $\gamma \varepsilon$ avant $\Delta$ tayópas.

L. 17 : $\dot{\varepsilon} \alpha v \tau \tilde{i}$ (ou $\dot{\varepsilon} \alpha v \tau \tilde{\omega}$ i- suggéré par Ziehen, si l'on admet la non transcription de l'iôta anekphônèton, comme dans le texte du règlement) nous semble moins

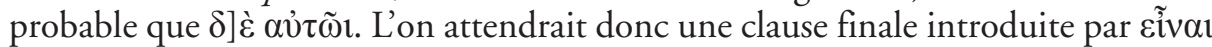
$\delta] \grave{\varepsilon} \alpha \hat{\tau} \tau \tilde{\omega} \mathrm{i}$, d'autant plus que, conformément à ce que montre le texte du règlement à droite, il est absolument nécessaire d'ajouter une dix-huitième ligne.

Et enfin, une remarque d'ensemble : quelle autre aurait pu être la raison pour graver sur deux colonnes sur la même plaque à la fois un décret (public, comme nous le verrons) et le règlement concernant l'achat de la prêtrise, finalement assumé par un certain Tí $\mu[\alpha 10 \varsigma] \Sigma \tau \rho \alpha ́ \tau \omega v o \zeta$, sinon que pour mettre en évidence les mérites de celui qui se chargea du culte des Dieux de Samothrace. Le décret aura été pris pour ce même citoyen.

Forts de tous ces points de départ et compte tenu du fait que certaines formules à insérer de manière quasiment obligatoire réclament des lignes plutôt longues - et de toute façon, plus longues que dans la colonne II -, nous proposons :

col. I

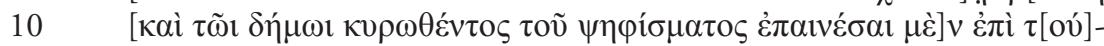

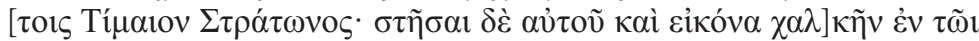

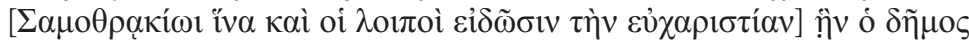

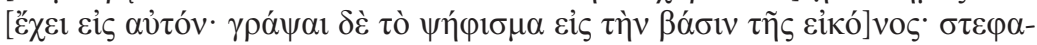

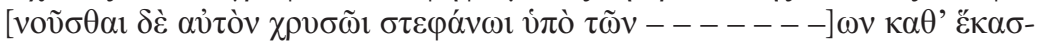

15 [

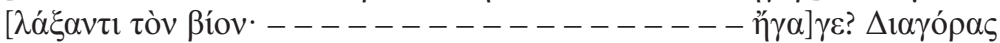

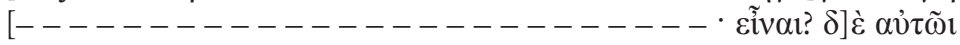
[-------------------------------] vacat 


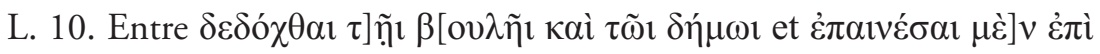
$\tau[$ ov́torc] il faut trouver quelque chose pour remplir la lacune. Nous suggérons $\kappa v \rho \omega-$

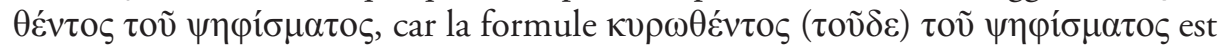
fréquente dans les inscriptions ${ }^{10}$ et figure aussi, sous diverses variantes, chez les auteurs,

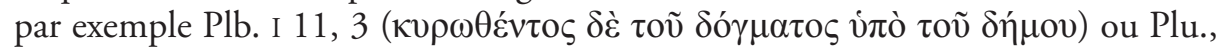

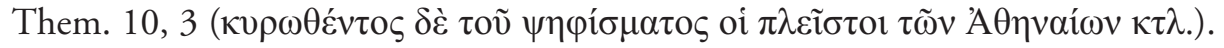

L. 12. Le restitution [ $\left.\Sigma \alpha \mu 0 \theta \rho \alpha \kappa^{\prime} \omega 1\right]$ s'impose au vu du contexe, bien que ce sanctuaire ne soit pas attesté à Tomis par d'autres inscriptions.

L. 12-13. À la fin de la 1. 12, nous ne voyons possible que ̣̣̂v ó $\delta \tilde{\eta} \mu \mathrm{os,} \mathrm{ce}$ qui suppose un accusatif féminin vers la fin de la lacune. Tout semble s'éclairer par l'introduction d'une formule dite " hortative ". Voir, entre autres, à Tomis même, le célèbre décret concernant la garde civique Syll. ${ }^{3} 731=\mathrm{I}$. Tomis 2, 1. 42-45 : îv $\alpha$

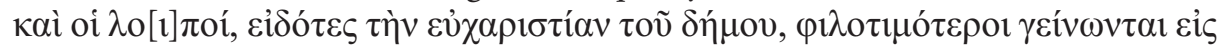

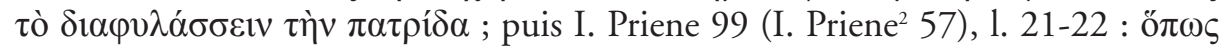

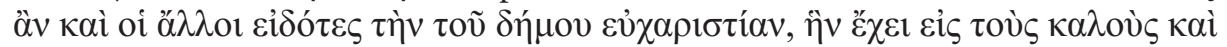

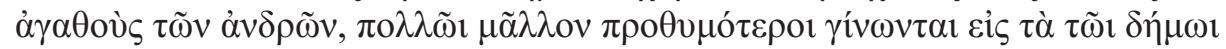

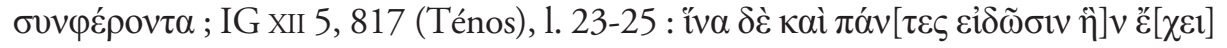

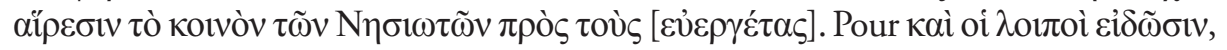
voir les formules suivies de ötı dans IG XII 7, 221b, 1. 22 (Amorgos) et I. Ephesos 1411,

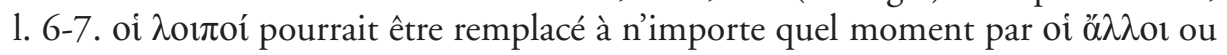

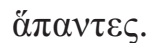

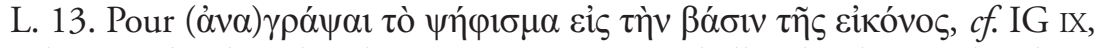
2, 489a (Phayttos de Thessalie), 1. 22 ; IG XII 9, 899 (Chalkis d’Eubée), col. c, 1. 5. L. 15. Voir, à titre d'exemple, OGIS 6 (Skepsis de Troade), 1. 30-31: $\alpha \pi \alpha \gamma-$

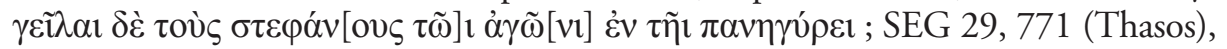

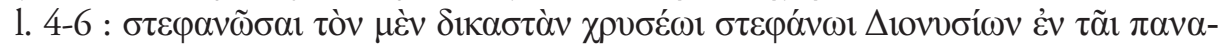

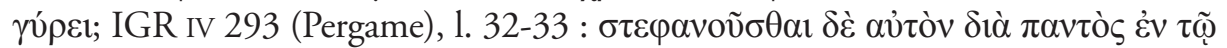

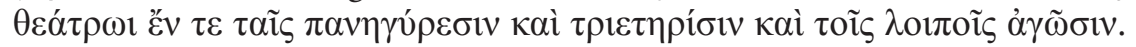

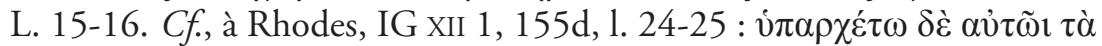

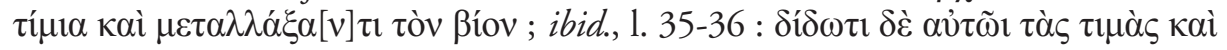

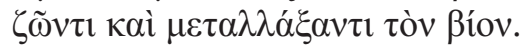

L. 16. La particule $\gamma \varepsilon$ étant plutôt improbable à notre avis, nous préférons

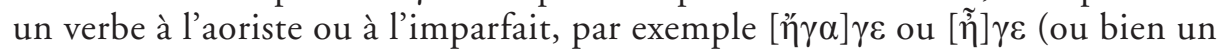
composé de ces verbes). La présence de la personne répondant au nom de $\Delta$ tayópas

${ }^{10}$ Une recherche menée sur PHI nous a fourni vingt occurrences dans le bassin égéen et sept

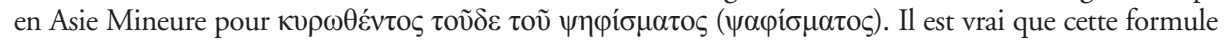

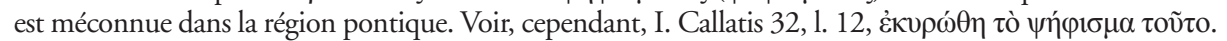

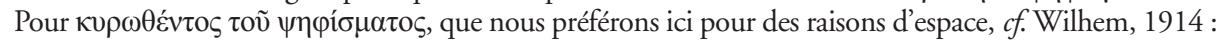
43-48, 1. 13-14 d'un décret d'Hyllarima de Carie. Voir aussi Robert, 1929 : 154-156 [= Robert, 1969, 129-131]. 
demeure énigmatique : est-ce le trésorier ou un autre magistrat censé mettre en œuvre l'une des décisions du décret?

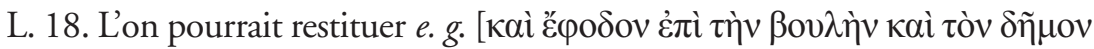

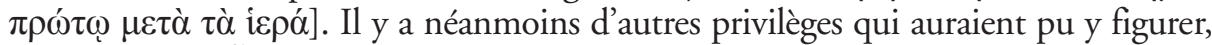

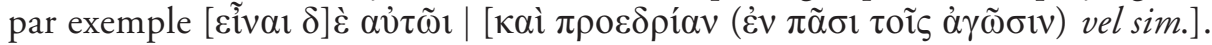
Il arrive même parfois que la cité offre au titulaire une somme pour accomplir le

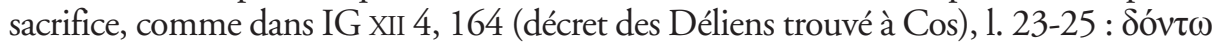

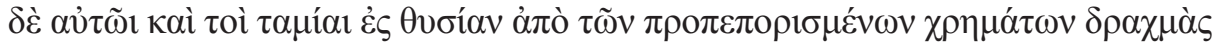
$\pi \varepsilon v \tau \eta \dot{\kappa o v} \tau \alpha$.

Malgré les doutes de D. M. Pippidi ${ }^{11}$, il s'agit donc d'un décret public. Selon toute probabilité, les deux documents furent gravés en même temps - à en juger d'après les traits paléographiques, autour de 100 a.C. - pour honorer Timaios, fils de Stratôn, bienfaiteur de la cité et prêtre des Grands Dieux de Samothrace. Il est pourtant impossible d'établir si les actes récompensés par le conseil et le peuple de Tomis étaient antérieurs à l'achat de la prêtrise. Les documents auraient pu être classés par ordre d'importance et non chronologique, pendant que la différence d'orthographe eût rendu compte des préférences des deux secrétaires.

\section{BIBLIOGRAPHIE}

I. Tomis Suppl. = A. AVram - M. BăRbUlesCU - L. BuZOIANU (2018): Inscriptions grecques et latines de Scythie Mineure, vol. VI. Suppléments, fasc. 2 : Tomis et son territoire, Bucarest - Paris.

BÂLTÂC, A. - ŞTIRBULESCU, C. - ŞTEFAn, A. [en collaboration avec ApOSTOL, V.] (2015) : Catalogul colecţiei lapidarium, vol. I. Piese greco-romane, Musée national d'histoire de la Roumanie, Bucarest.

Chiekova, D. (2008) : Cultes et vie religieuse des cités grecques du Pont Gauche (VII -Ir siècles avant J.-C.), Publications universitaires européennes, série XXXVIII, Archéologie, vol. 76, Peter Lang, Berne et autres.

Cole, S. G. (1984) : Theoi Megaloi: The Cult of the Great Gods at Samothrace, Etudes préliminaires aux religions orientales dans l'Empire romain, 96, Brill, Leyde.

DANOFF, C. M. (1932) : Die griechischen Inschriften aus Tomis und Kallatis, Diss. [thèse de doctorat inédite sous la direction de A. WILHELM], Vienne.

Delatte, A. (1955) : Le cycéon, breuvage rituel des mystères d'Éleusis, Les belles lettres, Paris.

Hemberg, B. (1950) : Die Kabiren, Almquist \& Wiksell, Uppsala.

Herbrecht, H. (1885) : De sacerdotii apud Graecos emptione venditione, Diss., Strasbourg.

Kern, O. (1919) : "Kabeiros und Kabeiroi ", RE x.2 : cols. 1399-1450.

Lupu, E. (2009) : Greek Sacred Law. A Collection of New Documents (2 éd.), Religions in the GraecoRoman World, 152, Brill, Leyde.

${ }^{11}$ Pippidi, $1967: 225$ [= Pippidi, $\left.1988: 224\right]$. 


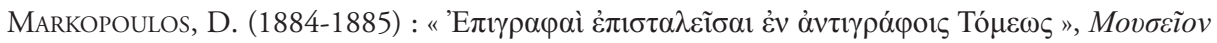

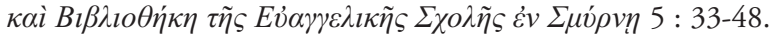

MicheL, Ch. (1900) : Recueil d'inscriptions grecques, H. Lamertin, Bruxelles.

NiLsson, M. P. (1955) : Geschichte der griechischen Religion, vol. I². Die Religion Griechenlands bis auf die griechische Weltherrschaft, Handbuch der Altertumswissenschaft, vol. v.2, C. H. Beck, Munich.

PARKer, R., OBbINK, D. (2000) : « Aus der Arbeit der ‘ Inscriptiones Graecae VI `. Sales of Priesthood on Cos I », Chiron $30: 415-449$.

PÂRVAN, V. (1924) : «Une nouvelle inscription de Tomi ", Dacia 1 : 273-279.

Pippidi, D. M. (1962) : " Pour une histoire des cultes d'Istros. Documents d'époque hellénistique », StudClas $4: 125-142$.

PIPPIDI, D. M. (1967) : «Tomitana iterum », StudClas 9 : 224-226.

PIPPIDI, D. M. (1969) : Studii de istorie a religiilor antice, Editura ştiinţifică, Bucarest.

PIPPIDI, D. M. (1988) : Studii de istorie şi epigrafie, Editura Academiei, Bucarest.

Poland, F. (1909) : Geschichte des griechischen Vereinswesens, Teubner, Leipzig.

ROBERT, L. (1929) : « Notes d'épigraphie hellénistique », BCH 53 : 151-165.

RoBerT, L. (1935-1936) : «Règlement religieux de Tomis », Istros 2 : 10-11.

ROBERT, L. (1966) : "Sur un décret d'Ilion et sur un papyrus concernant les cultes royaux ", dans Essays in Honor of C. Bradford Welles, American Studies in Papyrology, 1, American Society of Papyrologists, New Haven, pp. 175-211.

Robert, L. (1969) : Opera Minora Selecta, vol. I, A. M. Hakkert, Amsterdam.

Robert, L. (1990) : Opera Minora Selecta, vol. vII, A. M. Hakkert, Amsterdam.

RousseL, P. (1926) : «Remarques sur quelques règlements religieux », BCH 50 : 305-318.

Rubensohn, O. (1898) : «Kerchnos », $\operatorname{MDAI(A)} 23: 271-306$.

SOKOLOWSKI, F. (1969) : Lois sacrées des cités grecques, École française d'Athènes, Travaux et mémoires, 18, De Boccard, Paris.

STOIAN, I. (1962) : Tomitana. Contribuţii epigrafice la istoria cetăţii Tomis, Editura Academiei, Bucarest.

TocilesCu, G. (1882) : «Inschriften aus der Dobrudscha », Archäologisch-epigraphische Mitteilungen aus Österreich-Ungarn $6: 1-52$.

WilHelm, A. (1914) : Neue Beiträge zur griechischen Inschriftenkunde III, SBWien 175, 1, Vienne.

Ziebarth, E. (1896) : Das griechische Vereinswesen, S. Hirzel, Stuttgart. 